\title{
Root-associated microorganisms reprogram plant life history along the growth-stress resistance tradeoff
}

\author{
Mohammadhossein Ravanbakhsh $\mathbb{1}^{1} \cdot$ George A. Kowalchuk ${ }^{1}$ - Alexandre Jousset ${ }^{1,2}$
}

Received: 5 October 2018 / Revised: 30 June 2019 / Accepted: 9 August 2019 / Published online: 11 September 2019

(c) The Author(s), under exclusive licence to International Society for Microbial Ecology 2019

\begin{abstract}
Growth-defense tradeoffs are a major constraint on plant evolution. While the genetics of resource allocation is well established, the regulatory role of plant-associated microorganisms is still unclear. Here, we demonstrate that plantassociated microorganisms can reposition the plant phenotype along the same growth-defense tradeoff that determines phenotypic effects of plant mutations. We grew plants with microorganisms altering ethylene balance, a key hormone regulating plant investment into growth and stress tolerance. Microbial ethylene reduction had a similar effect to mutations disrupting ethylene signaling: both increased plant growth but at the cost of a strong stress hypersensitivity. We conclude that microbial impact on phenotype can offset the effects of mutations and that apparent plant growth promotion has strong pleiotropic effects. This study confirms that plant life history should be addressed as a joint product of plant genotype and its associated microbiota.
\end{abstract}

\section{Introduction}

Plants face multiple stressors during their life cycle. Balancing resource allocation between growth and stress resistance is therefore critical for optimizing ultimate fitness [1]. In plants, resource allocation into stress resistance is to a large extent coordinated by ethylene signaling [2]. Plants react to stress by upregulating ethylene, which triggers a range of physiological adaptations [3, 4]. These adaptations can help the plant cope with stressors, but they are also costly to implement. Direct metabolic costs or pleiotropic

Supplementary information The online version of this article (https:// doi.org/10.1038/s41396-019-0501-1) contains supplementary material, which is available to authorized users.

Mohammadhossein Ravanbakhsh

m.ravanbakhsh@uu.nl

$\triangle$ Alexandre Jousset

a.1.c.jousset@uu.nl

1 Ecology and Biodiversity Group, Institute of Environmental Biology, Utrecht University, Utrecht, The Netherlands

2 Jiangsu Provincial Key Lab for Organic Solid Waste Utilization, National Engineering Research Center for Organic-based Fertilizers, Jiangsu Collaborative Innovation Center for Solid Organic Waste Resource Utilization, Nanjing Agricultural University, Weigang 1, Nanjing 210095, PR China effects may cause tradeoffs between stress resistance and growth rate $[1,5]$. Optimal ethylene homeostasis is therefore important to reach an optimal phenotype that maximizes fitness under the given environmental constrains.

Mutations affecting hormonal signaling can have strong effects on the whole plant life history and can rapidly generate novel phenotypes. For instance, mutation of a single gene responsible for hormone-sensing can dramatically affect several traits ranging from germination to flowering and biomass production [2]. In addition to the plant genome, there is an increasing awareness that several plant traits are co-regulated by plant-associated microorganisms [6]. Given their impact on plant hormonal balance, microbe-mediated alterations of plant phenotype can be expected to generate fitness effects constrained by the same tradeoffs as plant mutations. However, to date, most research on such plant-microbe interactions has focused on single-trait approaches or on final plant productivity, often in the search for "beneficial" microbial traits. We propose that alterations of plant hormonal balance by microorganisms should not be considered inherently as beneficial or deleterious, but rather as ways to generate a new phenotype, whose success will depend on the given environmental conditions. The current study seeks to test this hypothesis by examining how microbial alterations in plant ethylene levels impact plant growth under stress versus nonstress conditions. 
Microorganisms impact plant ethylene signaling via several different mechanisms. For instance, auxinproducing microorganisms cause an increase in ethylene levels [7], while other microbes can reduce ethylene levels by producing the enzyme ACC (1-aminocyclopropane-1 carboxylic acid), which cleaves the direct precursor of ethylene [8]. Plant growth and stress tolerance are negatively correlated [9], and both are under the regulation of ethylene [4]. Given the core role of ethylene signaling and priming for stress resistance in plants [3, 4, 10], we propose that bacteria increasing ethylene will shift plant resource allocation toward more stress resistance, while those reducing it will lead to more relative investment into growth.

We examined the effect of microbial alteration of ethylene on plant growth along a gradient of cadmium stress, a heavy metal widespread in soil [11], that causes already at low concentration oxidative stress, photosynthesis crisis, and ultimately severe yield declines [10, 12]. We inoculated Arabidopsis thaliana with Pseudomonas putida UW4, a model bacterium able to degrade the ethylene precursor ACC, or an AcdS $^{-}$isogenic mutant of this strain, lacking the ability to reduce ethylene. We tracked plant growth in the absence or presence of cadmium stress, allowing us to define growth and stress resistance in each treatment. We then compared the effect of the inoculated microorganisms with the impacts of plant mutations that alter ethylene signaling: using the same growth conditions, we followed the development of wild-type $A$. thaliana Col-0 ecotype compared with an ethylene overproducer (etol) and an ethylene-insensitive mutant (ein3eill). Finally, we validated the importance of ethylene as underlying mechanism by treating plants with chemicals stimulating or blocking ethylene synthesis.

We examined the hypothesis that microbial modification of ethylene levels in plants shifts plant phenotype along the same growth-stress tolerance tradeoffs as caused by genetic variation in the plant genome. We expected that plant life history will be driven as much by the variation caused by root-associated microorganisms than by mutations in the plant genome itself.

\section{Method and materials}

\section{Plant materials}

We used Arabidopsis thaliana (L.) Heynh., ecotype Columbia (hereafter referred to as "Col-0") as a model plant. To validate the importance of ethylene signaling, we further used the ethylene overproducer mutant etol [13] and the ethylene-insensitive mutant ein3eill [14], two isogenic mutants of Col-0. All seeds were obtained from the Plant
Ecophysiology research group, Utrecht University, the Netherlands.

\section{Bacterial strains}

Pseudomonas putida UW4 wild-type strain (later "wildtype") and an isogenic ACC (1-aminocyclopropane-1-carboxylate) deaminase-deficient mutant (later "AcdS"”) were obtained from Prof. Glick, Department of Biology, University of Waterloo, Canada. Pseudomonas putida UW4 is as a model organism that has been used to study the role of ACC deaminase production in plant-microbe interactions [15]. The ACC deaminase-deficient mutant $\left(\mathrm{AcdS}^{-}\right)$was obtained by insertion of a tetracycline resistance gene into the ACC deaminase gene coding region [16]. Bacterial strains were kept as frozen stocks at $-80{ }^{\circ} \mathrm{C}$. Prior to experiments, one single colony was grown overnight on TSB medium (wild-type) or TSB and $100 \mu \mathrm{g} \mathrm{mL}^{-1}$ tetracycline $\left(\mathrm{AcdS}^{-}\right)$. Bacteria were harvested by centrifugation $(6000 \times g, 10 \mathrm{~min})$, washed three times with $10 \mathrm{mM} \mathrm{MgSO}_{4}$ and adjusted to a density of $10^{8}$ cells $\mathrm{mL}^{-1}$. Fifty microliter of bacterial suspension was inoculated to the base of each plant after transferring the seedling to a new pot (describe in "Pot experiment" section) or new agar plate medium (describe in "Agar plate assay" section).

\section{Model abiotic stressor}

We used cadmium to generate moderate to severe abiotic stress. Plants induce ethylene production and active signal transduction in response to cadmium stress [4, 10, 17]. The levels of cadmium for stress treatments were selected based on literature [18] to approximately match those commonly encountered in cadmium-contaminated agricultural soils.

\section{Experimental design}

We used two complementary experiments. In the main experiment, we used a pot experiment to evaluate the effects of bacterial modulation of ethylene signaling on plant growth and cadmium stress resistance at different levels of cadmium. To validate the results as product of ethylene signaling manipulation in the first complementary experiment, we selected a moderate level of cadmium $(50 \mu \mathrm{M})$ and compared the effects of bacterial inoculation on growth and stress resistance of Arabidopsis thaliana Col-0 with the effects of mutation in ethylene overproducing mutant (etol) or an ethylene perception impaired mutant (ein3eill). In a second experiment, we performed another validation of ethylene-induced life history strategy by growing axenic plants with or without cadmium on agar plates supplemented with chemicals stimulating or inhibiting ethylene synthesis. 


\section{Pot experiment}

Seeds were sterilized in an open microcentrifuge tube placed for $4 \mathrm{~h}$ under an airtight desiccator jar. A $250 \mathrm{ml}$ backer containing $100 \mathrm{ml}$ bleach and $3 \mathrm{ml}$ concentrated $\mathrm{HCl}$ solution placed in desiccator jar before seal it. After sterilization and stratification for 4 days at $4{ }^{\circ} \mathrm{C}$ in the dark, plant seeds were sown on $12 \mathrm{~cm}$ square petri dishes containing agar-solidified Murashige and Skoog medium supplemented with $0.5 \%$ sucrose. Petri dishes were transferred and positioned vertically in a growth chamber $\left(20^{\circ} \mathrm{C} ; 70 \%\right.$ relative humidity, 9-h photoperiod; $\left.200 \mu \mathrm{mol} \mathrm{m} \mathrm{m}^{-2} \mathrm{~s}^{-1} \mathrm{PPFD}\right)$. For the pot experiment, 10-day-old seedlings were transferred to pots containing a mixture of fine sand and perlite (both pasteurized for $45 \mathrm{~min}$ at $90^{\circ} \mathrm{C}$ ), saturated by a modified Hoagland nutrient solution [19] and placed in the same walk-in growth chamber. At day 21, 180 seedlings were selected based on uniformity of developmental stage and inoculated with bacterial strains (see the previous section). Pots were drenched with $50 \mathrm{ml}$ of deionized water or different amounts of cadmium from a stock solution ( $1 \mathrm{mM} \mathrm{CdSO}_{4}$ salt stock solution), resulting in approximately $0,10,50,100$, and $250 \mu \mathrm{M}$ water-extractable cadmium in the soil solution. We used a full factorial experimental design with twelve replicates per treatment (six replicates were used for ethylene measurement and six for other plant parameters). Plants were grown for an additional 14 days and the pots were randomized every 3 days.

\section{Root colonization by the inoculated bacteria}

We assessed the density of wild-type Pseudomonas putida UW4 and its isogenic AcdS $\mathrm{S}^{-}$mutant strain on plant roots immediately after harvest. Roots were gently shaken to remove adhering soil, after which bacteria were recovered by shaking the roots in $10 \mathrm{mM} \mathrm{MgSO}_{4}$ for $30 \mathrm{~min}$ at $200 \mathrm{~min}^{-1}$, sonicating them for $20 \mathrm{~s}$ and vortexing for $30 \mathrm{~s}$ prior to serial dilution on appropriate culture medium: the density of the wild-type strain was enumerated on DF agar (Table S1, composition of medium) [20] supplemented with ACC as the sole nitrogen source [21]. The $\mathrm{AcdS}^{-}$mutant strain was enumerated on DF agar supplemented with $\left(\mathrm{NH}_{4}\right)_{2} \mathrm{SO}_{4}$ as the nitrogen source and $100 \mu \mathrm{g} \mathrm{ml}{ }^{-1}$ tetracycline.

\section{Shoot ethylene measurements}

We measured the ethylene levels in plant shoots $24 \mathrm{~h}$ after cadmium exposure (on day 25). Six plant replicates were used for this assay. Shoots were carefully separated from the roots with a razor blade and aerial parts placed in serum vials $(50 \mathrm{ml})$. After $2 \mathrm{~h}$, a $1 \mathrm{ml}$ of gas sample was taken from each vial and injected into a Chrompack Packard gas chromatograph model 438 A with a Poropack Q column (length $100 \mathrm{~cm}$, packed to $0.34 \mathrm{~g} \mathrm{~cm}^{-3}$ ) at $60^{\circ} \mathrm{C}$ to measure ethylene concentration. Ethylene levels were expressed in $\mathrm{pL} g$ (fresh weight) ${ }^{-1} \mathrm{~h}^{-1}$ (see [22]). Plants were then collected to measure bacterial colonization and plant biomass.

\section{Comparison of bacterial and mutation effects on plant phenotype}

In order to benchmark the effect of microorganisms on ethylene signaling, we repeated the pot experiments at two stress level ( 0 and $50 \mu \mathrm{MCd})$, including functional mutants of $A$. thaliana. At each stress level, we used five plant treatments: A. thaliana Col-0, the ethylene overproducer mutant etol [13], the ethylene-insensitive mutant ein 3 eill [14], Col-0 inoculated with WT bacteria, and Col-0 inoculated with mutant bacteria. Pots were drenched with cadmium solution as described above at the four full leaves stage (25 days post germination). Plants were grown for an additional 14 days, after which plants were harvested, and shoot dry weight was measured as indicator of plan biomass production. Eight replicates were set up per treatment.

\section{Agar plate assays using Col-0, eto1, ein3eil1, and chemical inhibitor of ethylene oxidase}

To evaluate in detail the effects of ethylene production and signaling on plant growth, we transfer the 10-day-old seedling (seedling preparation as describe in "Pot experiment" section) of Arabidopsis thaliana Col-0, etol, and ein3eill genotypes on cadmium-treated solidified agar without the addition of bacteria. We evaluated the effects of ethylene biosynthesis inhibitor $(10 \mu \mathrm{M} \alpha$-aminoisobutyric acid, AIB; ACC oxidase inhibitor), ethylene precursor (5 $\mu \mathrm{M} \mathrm{ACC})$, and ethylene pretreatment (with $1 \mu \mathrm{LL}^{-1}$ ethylene-air gas mixture) [23] on plant biomass in the cadmium-treated or cadmium-free solidified agar. Uniform 10-day-old seedlings (prepared as mentioned above) were transferred to new agar-solidified Hoagland medium [24] containing either 0 (control) or $10 \mu \mathrm{M}$ cadmium. Eight plants were grown on each square plate and at least five replicate plates were assessed for each treatment. Plants were positioned vertically in a walk-in growth chamber for an additional 10 days, after which plant shoot and root biomass were measured.

\section{Growth and stress resistance of wild-type plants inoculated with bacteria}

Plant tolerance to cadmium was defined as the biomass ratio between stress and control treatments (Normalized stress resistance). A tolerance index of 1 means that the plant is not affected by cadmium, an index of 0 indicates no growth in presence of cadmium.

The relative growth of plant mutants, bacterial treatments, and ethylene chemical modifiers (see next section) 
was all related to the normalized growth of Col-0 (i.e., with Col-0 growth at a value of 1, Fig. 4). We considered the biomass reduction by cadmium as an indication of a stress response in plant genotypes (Col-0, etol, and ein3eill) in both soil and agar plate experiment. The same calculations were done for plant growth and stress tolerance in Col-0 inoculated with bacterial treatment (no bacteria, wild-type bacteria, or $\mathrm{AcdS}^{-}$mutant bacteria) or Col-0 treated with ethylene chemical modifier (ACC oxidase inhibitor (AIB), ACC treatment, or ethylene pretreatment). This data normalization allowed us to compare all the results by assessing how different treatments shifted plant phenotype along the growth-stress tolerance tradeoff.

\section{Statistical analyses}

We used two-way ANOVAs to evaluate the interactive effects of cadmium concentration (factors; five levels in pot experiment and two levels on the in vitro experiments) and plant treatment by bacteria (three levels: non-bacterized control, wild-type, and $\mathrm{AcdS}^{-}$mutant bacterial treatment) on shoot ethylene level, shoot and root dry weight for different experimental setups. For the experiment on the plant genetic background, we evaluated the interactive effects of plant genotypes (three levels: Col-0, etol, and ein3eill) and cadmium treatments $(0$ and $50 \mu \mathrm{M})$ on shoot dry weight. When necessary, plant growth data were square-root transformed prior to analysis to meet the assumptions of normality and homoscedasticity. Similar statistical analyses were also performed for the in vitro plant assays for ethylene-modulating chemical treatments. We assessed the correlation between root dry weight and shoot ethylene level using separate one-way ANOVAs (separate in control and cadmium-treated condition). In order to dive deeper into the effect of ethylene reduction on plant stress tolerance, we calculated for each stress level the weight difference between plants treated with wild-type and $\mathrm{AcdS}^{-}$ bacteria (which we used as a proxy for the net effects of ACC deaminase enzyme).
In order to summarize the results of these three experiments and show the general interplay between growth and stress tolerance in the presence and absence of cadmium, we normalized plant growth in each treatment to the one of uninoculated and chemically untreated Col- 0 plants subjected to the corresponding stress treatment. This allowed us to examine if all results fit within a unified, overarching growth-stress resistance tradeoff (Fig. 4). All analyses were performed in SPSS (V. 22).

\section{Results}

\section{Effects of bacterial inoculation and cadmium level on plant ethylene level}

Both bacterial treatment $\left(F_{2,75}=52.65, p<0.0001\right)$ and cadmium concentration $\left(F_{4,75}=88.80, p<0.0001\right)$ significantly impacted ethylene levels in Arabidopsis thaliana. Ethylene concentrations increased with increasing cadmium level in the soil. However, it decreased for the most extreme cadmium contamination treatments (100 and $250 \mu \mathrm{M})$ (Fig. S1). The effect of cadmium on plant ethylene level was further modulated by bacterial inoculation and the genetic background of the introduced bacteria (Bacterial treatmentx cadmium level; $F_{8,75}=12.96, p<0.0001$ ). Inoculation with the wild-type bacterial strain on average reduced shoot ethylene significantly from 401 to $351 \mathrm{pL} \mathrm{g}^{-1}$ fresh weight $\mathrm{h}^{-1}$ compared with the no-bacteria control in cadmiumspiked soils. However, inoculation with the $\mathrm{AcdS}^{-}$mutant bacteria increased ethylene concentration, resulting in 524 $\mathrm{pL} \mathrm{g}^{-1}$ fresh weight $\mathrm{h}^{-1}$ in this treatment (Fig. S1).

\section{Shoot and root fresh weight and dry weight in gradient assay}

Fresh and dry weight of A. thaliana significantly decreased with increasing cadmium (Table 1, main effects of cadmium levels). At the highest cadmium concentration $(250 \mu \mathrm{M})$, the
Table 1 Two-way ANOVA table summarizing the interactive effects of cadmium treatment levels $(0,10,50,100$, and $250 \mathrm{mM}$ ), bacterial treatment (wild-type ACC deaminaseproducing bacteria, its isogenic ACC deaminase-deficient mutant, and no-bacterial control), and their interaction on plant fresh, dry weight, and shoot ethylene concentration in A. thaliana. One linear model was fitted separately for each dependent variable

\begin{tabular}{|c|c|c|c|c|c|c|c|c|}
\hline & & \multirow[b]{2}{*}{$D f$} & \multicolumn{2}{|c|}{ Fresh weight } & \multicolumn{2}{|c|}{ Dry weight } & \multicolumn{2}{|c|}{ Ethylene Conc. } \\
\hline & & & $F$ & $P$ & $F$ & $P$ & $F$ & $P$ \\
\hline \multirow[t]{4}{*}{ Shoot } & Cadmium levels & 4 & 244.144 & $<0.0001$ & 78.34 & $<0.0001$ & 335.77 & $<0.0001$ \\
\hline & Bacteria & 2 & 0.104 & 0.90 & 4.48 & 0.002 & 55.04 & $<0.0001$ \\
\hline & Cadmium $\times$ Bacteria & 8 & 4.115 & $<0.0001$ & 0.75 & 0.61 & 8.38 & $<0.0001$ \\
\hline & Error & 75 & & & & & & \\
\hline \multirow[t]{4}{*}{ Root } & Cadmium levels & 4 & 121.22 & $<0.0001$ & 210.33 & $<0.0001$ & & \\
\hline & Bacteria & 2 & 2.92 & 0.06 & 6.31 & 0.003 & & \\
\hline & Cadmium $\times$ Bacteria & 8 & 2.75 & 0.01 & 6.97 & $<0.0001$ & & \\
\hline & Error & 75 & & & & & & \\
\hline
\end{tabular}


plants showed serious symptoms of toxicity, even in the presence of bacteria. The effects of bacterial inoculation on the shoot and root dry weight were significant (Table 1, main effects of bacteria inoculation). The effects of bacteria on plant growth were further modulated by the cadmium concentration (Cadmium $\times$ Bacteria interaction, Table 1). As bacteria may influence plants in several ways, we used the dry weight difference between plants inoculated with the wild-type versus the AcdS ${ }^{-}$mutant bacteria as a measure of the effect of ACC deaminase enzyme: in the absence of stress, ACC deaminase-producing bacteria increased plant weight compared with the AcdS ${ }^{-}$mutant (Fig. 1). However, this effect switched to become negative under cadmium stress (Fig. 1). This result is in line with plant physiology studies demonstrating the importance of ethylene as a stress response-regulating hormone $[3,10]$. It further shows that ACC deaminase-producing bacteria may increase plant growth in the absence of stress, but decrease it in the presence of abiotic stressors such as cadmium.

Analysis of resource allocation further revealed a negative correlation between shoot ethylene concentration and root dry weight $\left(F_{1,17}=5.01, p=0.04, R^{2}=0.24\right)$ and total plant dry weight (marginally significant, $F_{1,17}=3.94, p=$ $0.06, R^{2}=0.22$ ) in the absence of stress (Fig. 2a). This result is in line with previously described effects of ethylene [25] and points to a central role of ethylene as a global (a)

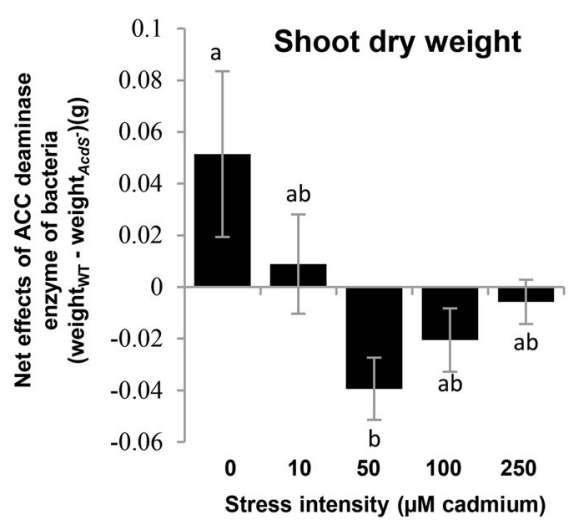

(b)

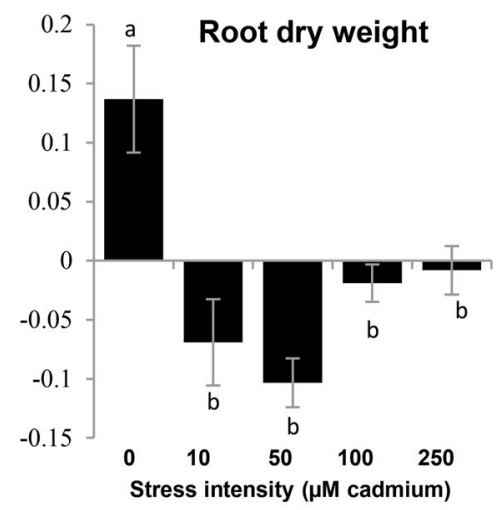

(c)

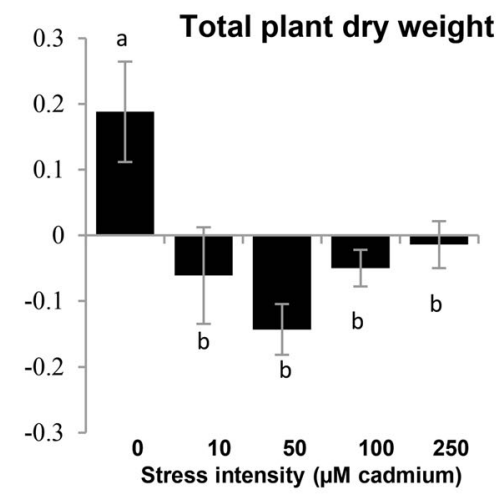

Fig. 1 Net effects of ethylene reduction by root-associated microbiota on plants growth along a stress gradient. The net effect was defined as the difference in shoot (a), root (b), and total dry weight (c) between Arabidopsis thaliana Col-0 plants inoculated with wild-type Pseudomonas putida UW4 or an isogenic ACC deaminase-deficient mutant. A positive net effect indicates that ACC deaminase-mediated reduction

(a)

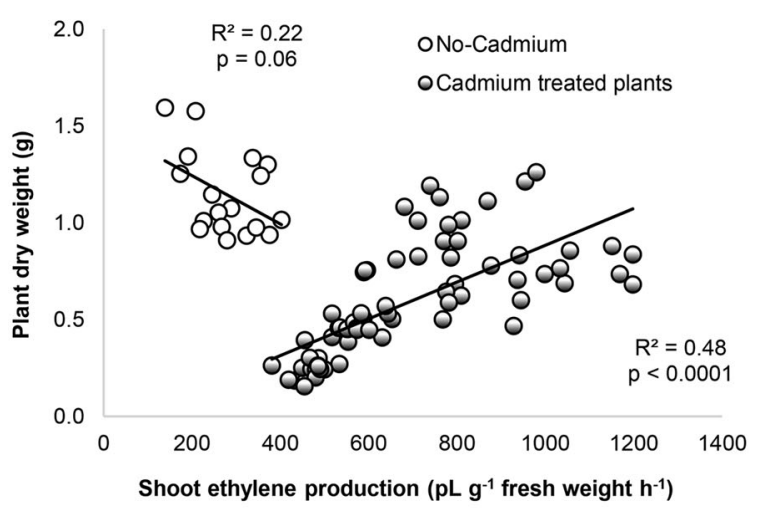

Fig. 2 Ethylene reduction by microorganisms impairs plant growth under abiotic stress. a High ethylene level is positively correlated with biomass production in A. thaliana under cadmium stress, but not in stress-free control, highlighting the importance of this hormone for stress tolerance. b Reduction of ethylene levels by ACC deaminaseproducing rhizosphere bacteria is correlated with a reduction of plant of plant ethylene increases plant biomass; a negative effect indicates an inhibition. Differences between treatments were analyzed using oneway ANOVAs of difference (wild-type bacteria subtracted mutant). Letters above each bar indicate statistically significant differences based on Tukey multiple range tests $(p<0.01)$. Error bars show \pm SE. The numbers of replicates were 6

(b)

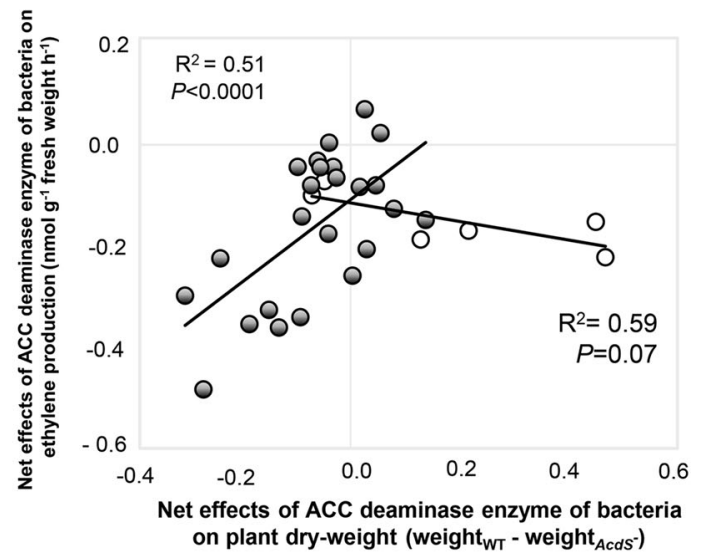

biomass under cadmium stress, but does not affect plant growth in the stress-free treatment. The net effects of ACC deaminase production are estimated as the difference in plant weight or ethylene level between plants inoculated with the wild-type, ACC deaminase-producing Pseudomonas putida UW4, and its isogenic $\mathrm{AcdS}^{-}$mutant. The numbers of replicates were 6 
regulator of plant resource allocation in addition to its role as a stress response regulator. Under cadmium stress conditions, shoot ethylene level in A. thaliana was positively correlated with both root dry weight $\left(F_{1,71}=65.42, p<\right.$ $\left.0.0001, R^{2}=0.49\right)$ and total plant dry weight $\left(F_{1,71}=66.62\right.$, $p<0.0001, R^{2}=0.48$; Fig. 2a). Further, reduction of ethylene levels by ACC deaminase-producing rhizosphere bacteria showed the same effects on plant biomass under cadmium stress and cadmium-free condition (Fig. 2b), indicating that differences in plant growth observed between the different bacterial and plant genetic backgrounds could be explained by variations in ethylene concentration.

\section{Bacterial colonization}

The density of recovered root-associated bacteria per $g$ root fresh weight decreased slightly along with cadmium gradient $\left(F_{4,75}=2.17, p=0.08\right)$. The densities of recovered wild-type ACC deaminase-producing bacteria and ACC deaminase-deficient mutant $\left(\mathrm{AcdS}^{-}\right)$were similar $\left(F_{2,75}=\right.$ $1.59, p=0.21)$, indicating that the lack of ACC deaminase did not affect bacterial fitness under the tested conditions.

\section{Effect of mutations altering ethylene signaling on growth and stress tolerance in Arabidopsis thaliana}

In order to confirm the importance of ethylene reduction as a mechanism linking ACC deaminase to plant growth and hypersensitivity to stress, we assessed the biomass production of sterile A. thaliana Col-0 (WT, normal ethylene signaling), etol (ethylene overproducer) and ein3eill (ethylene insensitive) growing at 0 (no stress) and $50 \mu \mathrm{M}$ water-soluble cadmium in soils (stress) (Fig. 3; Table S2, supporting information).

In the absence of stress, the ein3eill mutant showed higher biomass production compared with Col-0 (Relative growth of ein3eill compared with Col-0, Fig. 4). This mutant also harbored a higher sensitivity to stress, with cadmium causing a biomass reduction of $38 \%$ relative to the no-stress treatment, in comparison with a $14 \%$ reduction for Col-0, and a 9\% reduction for etol (Fig. 3; Table S2, supporting information). In contrast, ethylene overproducer mutant etol show a lower biomass production compared with Col-0 and ein3eill under the no-cadmium condition, but showed a higher tolerance to cadmium stress (Fig. 3). Together, these results indicate the importance of ethylene signaling as switch for resource allocation from growth to stress resistance.

\section{Effects of chemical ethylene manipulation on plant growth and stress resistance}

Shoot and root dry weight of A. thaliana significantly decreased in cadmium-treated agar plates, and the different chemical ethylene modifiers significantly altered these factors in plants (Table S3, supporting materials). Under the control condition (no-cadmium treatment), the ein3eill mutant and Col-0 treated with the ethylene biosynthesis inhibitor AIB both showed a significantly higher shoot (Fig. 4) and root dry weight compared with the untreated Col-0 (S2, supporting materials). Treatment of plants with ACC or pretreatment with ethylene decreased Col-0 dry weight in the control treatment but increased tolerance to cadmiums stress. Overall, the untreated Col-0 plants showed an intermediate phenotype, both in terms of growth

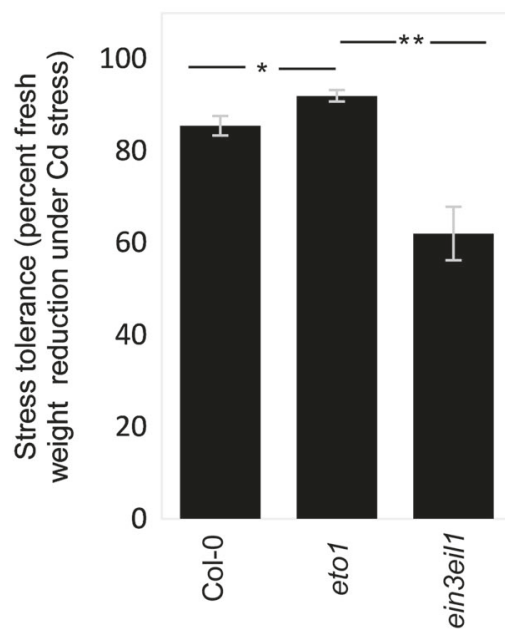

Fig. 3 Cadmium stress tolerance in Col-0, eto1, and ein3eil1 genotypes growing in $50 \mu \mathrm{M}$ cadmium compared with a no-cadmium treatment. ein3eill mutant showed $38 \%$ reduction in dry weight compared with $14 \%$ reduction in Col-0, and 9\% in etol. The Differences between Col-0,

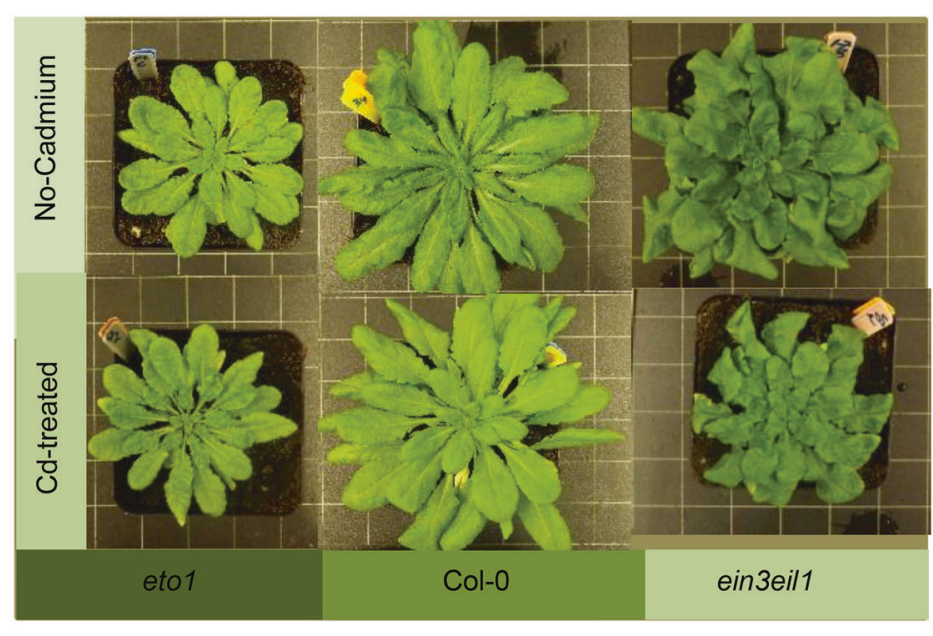

eto1, and ein3eill genotypes were analyzed by two-way ANOVA of plants and cadmium treatment $(0$ and $50 \mu \mathrm{M})$ (Table S2). Significant effects are shown by asterisks $(* p<0.05, * * p<0.01)$. Error bars show \pm SE. The numbers of replicates were 8 


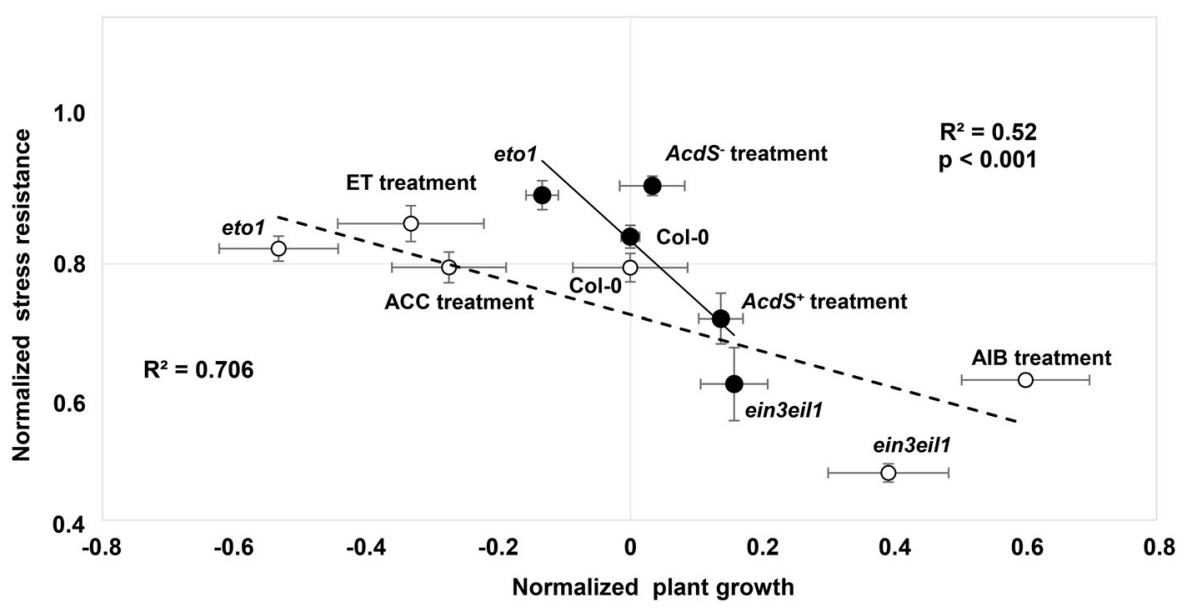

Fig. 4 Alterations of plant ethylene levels by microorganisms shifts Arabidopsis thaliana along growth-stress resistance tradeoffs. Growth and stress resistance in each treatment were normalized to Col-0 without microbial or chemical amendment as baseline. The $X$-axis shows plant growth, defines as shoot dry weight in the control, stressfree treatment. The $Y$-axis shows plant stress tolerance, defined as the ratio of shoot dry weight under cadmium stress relative to the control, stress-free treatment. Black line and closed symbols: pot experiment: Arabidopsis thaliana wild-type (Col-0) was inoculated with the ACC deaminase-producing Pseudomonas putida UW4, which reduces

and stress resistance, relative to the treatments resulting in increased or decreased ethylene levels.

\section{The growth-stress tolerance tradeoff in A. thaliana is the same for mutations- and microorganism- induced phenotypic changes}

In order to visualize whether phenotypic changes induced by ethylene manipulation by microbes, mutations, and chemicals are constrained by the same tradeoffs, we plotted for each treatment (two bacteria, three genotypes, and four chemicals) biomass production versus stress resistance. Inhibition of ethylene oxidase in Col-0 by AIB, or insensitivity as in the ein3eill mutant, showed the same response on solidified agar medium, namely increasing the biomass under control conditions, but leading to hypersensitivity to cadmium-induced stress. In contrast, ein3eill and the AIB treatment yielded greater a reduction of plant biomass under cadmium stress (Fig. 4). The same trends were observed for plant root weight in agar plate medium (Fig S2).

\section{Discussion}

Plants resource allocation between growth and stress resistance is a major evolutionary constrain on plants. As stress adaptation is costly, plant fitness will be constrained by growth-stress resistance tradeoffs [1]. In plants, resource allocation to stress resistance is to a large extent coordinated ethylene (Col-0 and AcdS ${ }^{+}$) or its isogenic ACC deaminase-deficient mutant, which causes an increase in plant ethylene ( $\mathrm{Col}-0$ and $\left.\mathrm{AcdS}^{-}\right)$. The effects of bacteria on plant growth and stress resistance are benchmarked to the most extreme available plant mutants leading to ethylene overproduction (etol) or in the opposite virtually complete ethylene insensitivity (ein3eill). Open symbols and dashed lines: results of an axenic experiment in which plant ethylene was manipulated by pretreatment with ethylene, its precursor ACC or the inhibitor AIB. Error bars show \pm SE

by ethylene signaling. As this hormone is strongly affected by plant-associated microorganisms [26], we postulated that microbes and genetic mutations may have similar effects on plant life history. In this study, we demonstrate that phenotypic effects of bacteria altering plant ethylene levels are of the same magnitude as those caused by mutation in the plant genome. Further, phenotypes caused by mutations, microorganisms, or direct chemical alteration of ethylene levels in plants were all placed along the same growth-stress resistance tradeoff.

This study contributes to resolving an important paradox related to current paradigms in plant-microbe interactions and plant physiology: bacteria harboring ACC deaminase have widely been proposed as beneficial to plants $[8,27,28]$, yet plant physiology studies consistently predict that lower ethylene levels will make plants more sensitive to stress $[4,10]$. We propose that this apparent contradiction can be harmonized if one takes plant life history tradeoffs into account. Tradeoffs emerge when investment in one potentially adaptive trait reduces the ability to develop alternative potentially adaptive traits. It has been well established that investment into growth diverts resources from defense mechanisms and vice versa [1]. In this context, any change in hormonal balance will not be beneficial or deleterious per se, but will instead cause a shift along existing tradeoffs.

We show that ethylene reduction by mutation, chemical inhibitors, or microbiota increases plant growth under stress-free conditions (Fig. 4), but also comes at a cost of a 
hypersensitive response to stress. This effect was directly proportional to the stress level imposed. In absence of stress, ethylene concentrations negatively correlate with plant biomass production, suggesting that plants with a higher ethylene concentration invest less into vegetative growth. Accordingly, reduction of ethylene leads to an increase in biomass. In the presence of a moderate stress, however, the situation is reversed. In this case, ethylene levels positively correlate with biomass, highlighting the importance of this hormone as a coordinator of physiological adaptations that allow the plant to cope with stressors. In line with this observation, the reduction of ethylene levels under stressful conditions causes a decrease in plant performance. Finally, as stress intensity increases and goes beyond the physiological adaptation potential of plants, ethylene ceases to be relevant. Plants are inhibited in all cases, and alterations of ethylene signaling no longer significantly impact plant growth.

A key finding of this study is that ACC deaminase production by bacteria outside the plant create such a strong sink for ACC that it reduces ethylene concentration and plant stress tolerance to a similar degree as the complete shutdown of ethylene signaling by mutations in the plant genome (einleil3) or the chemical inhibition of ethylene synthesis (AIB). In contrast, the $\mathrm{AcdS}^{-}$bacterial mutant, which indirectly induce ethylene production by producing auxin [7], can shift the plant toward a similar stresstolerance level achieved by the ethylene overproducer plant mutant etol. It is important to note that the enzyme ACC deaminase enzyme directly contributes to bacterial fitness by allowing them to use ACC as a source of nitrogen. This may cause a potential conflict of interest between plants, who depend on high ethylene levels for their survival under adverse conditions, and microbes, who have a direct interest in consuming ACC. This may have broad implications for plant evolution: as the effect of mutations in the plant genome may be smaller than the effects due to variation in the plant microbiome, microbial co-regulation of ethylene may either block evolution or in contrast generate novel evolutionary trajectories [26].

We conclude that microbial manipulation of plant ethylene signaling (and potentially of other hormones) is not a plant growth-promoting trait per se, and we call for a reconsideration of the standard plant-microbe interactions concept. Instead of being "beneficial" or "deleterious", we propose to approach microbiota as modulators of plant phenotype, helping plants to explore the potential phenotypic space offered by their genetic background. Microbiota may thus have an effect on plant phenotype that bears strong similarities with breeding, creating new phenotypes by reshuffling existing traits. The idea of an holobiont-level regulation of plant phenotype has been proposed to drive plant fitness $[6,26]$ but was to date difficult to demonstrate experimentally. Here, we prove that single mutations in root-associated microorganisms are as much a driver of plant phenotype as mutations in the plant genome. We, therefore, conclude that both plants and the microbiome should include in the future studies in plant ecology and evolution.

Acknowledgements Laurentius A.C.J. Voesenek and Rashmi Sasidharan are acknowledged for their contribution to the project design. The authors would like to thank Prof. Bernard Glick, Department of Biology, University of Waterloo, Waterloo, ON, Canada for providing the bacteria strains. Francisco X Nascimento from the plant-microbe interaction of Utrecht University is acknowledged for his inputs for experimental setup on the agar plate. Sjon Hartman from the ecophysiology group of Utrecht University is acknowledged for his inputs in ethylene pretreatment. Gerrit Rouwenhorst from Ecology and Biodiversity group, Utrecht University is acknowledged for his valuable advice for plant and soil digestions and doing ICP measurements. Rob Welschen from the Plant Ecophysiology group, Peter Veenhuizen, G.P. Verduyn, and Paolo Carril Vaglini from the Ecology and Biodiversity group, Utrecht University are acknowledged for their technical assistance and advice.

Author contribution MR, AJ, and GK designed the experiment. MR collected the data, performed lab work, and analyzed output data. MR and AJ wrote the first draft of the manuscript, and all authors contributed substantially to revisions and final approval.

\section{Compliance with ethical standards}

Conflict of interest The authors declare that they have no conflict of interest.

Publisher's note: Springer Nature remains neutral with regard to jurisdictional claims in published maps and institutional affiliations.

\section{References}

1. Huot B, Yao J, Montgomery BL, He SY. Growth-defense tradeoffs in plants: a balancing act to optimize fitness. Mol Plant. 2014;7:1267-87.

2. Roux F, Touzet P, Cuguen J, Le Corre V. How to be early flowering: an evolutionary perspective. Trends Plant Sci. 2006;11:375-81.

3. Sasidharan R, Voesenek LACJ. Ethylene-mediated acclimations to flooding stress. Plant Physiol. 2015;169:3-12.

4. Thao NP, Khan MIR, Thu NBA, Hoang XLT, Asgher M, Khan NA, et al. Role of ethylene and its cross talk with other signaling molecules in plant responses to heavy metal stress. Plant Physiol. 2015;169:73-84.

5. Gudelj I, Weitz JS, Ferenci T, Claire Horner-Devine M, Marx CJ, Meyer JR, et al. An integrative approach to understanding microbial diversity: from intracellular mechanisms to community structure. Ecol Lett. 2010;13:1073-84.

6. Vandenkoornhuyse P, Quaiser A, Duhamel M, Le Van A, Dufresne A. The importance of the microbiome of the plant holobiont. New Phytol. 2015;206:1196-206.

7. Ravanbakhsh M, Sasidharan R, Voesenek LACJ, Kowalchuk GA, Jousset A. ACC deaminase-producing rhizosphere bacteria modulate plant responses to flooding. J Ecol. 2017;105:979-86.

8. Glick BR. Bacteria with ACC deaminase can promote plant growth and help to feed the world. Microbiol Res. 2014;169:30-39. 
9. Araújo WL, Fernie AR, Nunes-Nesi A. Control of stomatal aperture. Plant Signal Behav. 2011;6:1305-11.

10. Keunen E, Schellingen K, Vangronsveld J, Cuypers A. Ethylene and metal stress: small molecule, big impact. Front Plant Sci. 2016;7:23.

11. Reimann C, Birke M, Demetriades A, Filzmoser P, O'Connor P. Chemistry of Europe's agricultural soils, part A. https://www. schweizerbart.de/publications/detail/isbn/9783510968466. 2014;102: p. 880.

12. Mendelssohn IA, McKee KL, Kong T. A comparison of physiological indicators of sublethal cadmium stress in wetland plants. Environ Exp Bot. 2001;46:263-75.

13. Wang KL-C, Yoshida H, Lurin C, Ecker JR. Regulation of ethylene gas biosynthesis by the Arabidopsis ETO1 protein. Nature. 2004;428:945.

14. Alonso JM, Stepanova AN, Solano R, Wisman E, Ferrari S, Ausubel FM, et al. Five components of the ethyleneresponse pathway identified in a screen for weak ethyleneinsensitive mutants in Arabidopsis. Proc Natl Acad Sci. 2003; 100:2992-7.

15. Duan J, Jiang W, Cheng Z, Heikkila JJ, Glick BR. The complete genome sequence of the plant growth-promoting bacterium Pseudomonas sp. UW4. PLOS ONE. 2013;8:e58640.

16. Li J, Ovakim DH, Charles TC, Glick BR. An ACC deaminase minus mutant of Enterobacter cloacae UW4 no longer promotes root elongation. Curr Microbiol. 2000;41:101-5.

17. Ravanbakhsh M, Kowalchuk GA, Jousset A. Optimization of plant hormonal balance by microorganisms prevents plant heavy metal accumulation. J Hazard Mater. 2019;379:120787.

18. Remans T, Thijs S, Truyens S, Weyens N, Schellingen K, Keunen E, et al. Understanding the development of roots exposed to contaminants and the potential of plant-associated bacteria for optimization of growth. Ann Bot. 2012;110:239-52.

19. Smeets K, Ruytinx J, Semane B, Van Belleghem F, Remans T, Van Sanden S, et al. Cadmium-induced transcriptional and enzymatic alterations related to oxidative stress. Environ Exp Bot. 2008;63:1-8

20. Dworkin M, Foster JW. Expriment with some micronutrients which utilize ethylene and hydrogen. J Bacteriol. 1958;75:592.

21. Donna M. Penrose, Bernard R. Glick. Methods for isolating and characterizing ACC deaminase-containing plant growth-promoting rhizobacteria. Physiologia Plantarum 2003;118:10-5.

22. Voesenek LACJ, Jackson MB, Toebes AHW, Huibers W, Vriezen WH, Colmer TD. De-submergence-induced ethylene production in Rumex palustris: regulation and ecophysiological significance. Plant J Cell Mol Biol. 2003;33:341-52.

23. Veen H, van, Mustroph A, Barding GA, Eijk MV, WelschenEvertman RAM, Pedersen O, et al. Two rumex species from contrasting hydrological niches regulate flooding tolerance through distinct mechanisms. Plant Cell Online. 2013;25:4691-707.

24. Hoagland DR, Arnon DI. Growing plants without soil by the water-culture method. Grow Plants Soil Water-Cult Method. 1938;347:1-39.

25. Kieber JJ, Rothenberg M, Roman G, Feldmann KA, Ecker JR. CTR1, a negative regulator of the ethylene response pathway in arabidopsis, encodes a member of the Raf family of protein kinases. Cell. 1993;72:427-41.

26. Ravanbakhsh M, Sasidharan R, Voesenek LACJ, Kowalchuk GA, Jousset A. Microbial modulation of plant ethylene signaling: ecological and evolutionary consequences. Microbiome. 2018;6:52.

27. Safronova VI, Stepanok VV, Engqvist GL, Alekseyev YV, Belimov AA. Root-associated bacteria containing 1-aminocyclopropane-1carboxylate deaminase improve growth and nutrient uptake by pea genotypes cultivated in cadmium supplemented soil. Biol Fertil Soils. 2006;42:267-72.

28. Zhang Y, He L, Chen Z, Wang Q, Qian M, Sheng X. Characterization of ACC deaminase-producing endophytic bacteria isolated from copper-tolerant plants and their potential in promoting the growth and copper accumulation of Brassica napus. Chemosphere. 2011;83:57-62. 